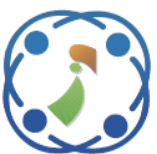

INASS
International Journal of

Intelligent Engineering \& Systems

http://www.inass.org/

\title{
Engagement Detection through Facial Emotional Recognition Using a Shallow Residual Convolutional Neural Networks
}

\author{
Michael Moses Thiruthuvanathan ${ }^{1 *}$ \\ Balachandran Krishnan ${ }^{1}$ \\ Madhavi Rangaswamy² \\ ${ }^{I}$ Department of Computer Science and Engineering, School of Engineering and Technology, \\ CHRIST (Deemed to be University), Bangalore, India \\ ${ }^{2}$ Department of Psychology, School of Social Sciences, CHRIST (Deemed to be University), Bangalore, India \\ * Corresponding author's Email: Michael.moses@christuniversity.in
}

\begin{abstract}
Online teaching and learning has recently turned out to be the order of the day, where majority of the learners undergo courses and trainings over the new environment. Learning through these platforms have created a requirement to understand if the learner is interested or not. Detecting engagement of the learners have sought increased attention to create learner centric models that can enhance the teaching and learning experience. The learner will over a period of time in the platform, tend to expose various emotions like engaged, bored, frustrated, confused, angry and other cues that can be classified as engaged or disengaged. This paper proposes in creating a Convolutional Neural Network $(\mathrm{CNN})$ and enabling it with residual connections that can enhance the learning rate of the network and improve the classification on three Indian datasets that predominantly work on classroom engagement models. The proposed network performs well due to introduction of Residual learning that carries additional learning from the previous batch of layers into the next batch, Optimized Hyper Parametric (OHP) setting, increased dimensions of images for higher data abstraction and reduction of vanishing gradient problems resulting in managing overfitting issues. The Residual network introduced, consists of a shallow depth of 50 layers which has significantly produced an accuracy of $91.3 \%$ on ISED \& iSAFE data while it achieves a 93.4\% accuracy on the Daisee dataset. The average accuracy achieved by the classification network is 0.825 according to Cohens Kappa measure.
\end{abstract}

Keywords: Student engagement detection, Residual networks, Convolutional neural network, Emotion detection, Facial expression recognition.

\section{Introduction}

Recognition of user interaction becomes highly important in a digital environment that is filled with information of consumers. It is important for applications to be "aware" of the user's presence when delivering information. Affect is a psychological process used to define the feeling and its external presentation. Affective computing aims at designing systems and tools capable of detecting, reading and simulating human effects across various sources such as face, speech and biological signals. Due to the availability of online resources to learn during these pandemic times, students are involved in attending online classes. Virtual learners take part in numerous instructional events including reading, writing, viewing video lessons, virtual exams and online meetings. They display different degrees of interaction during involvement in these educational events, such as fatigue, annoyance, excitement, indifference, uncertainty, and advantage in learning. To provide customized pedagogical support through online learner initiatives, it is critical that online educators reliably and effectively detect the state of involvement of their online learners. In the sense of online learning this paper provides a study of the state of the art in interaction identification. While students take part in these online classes, computing is required to estimate the valence and arousal to analyse the engagement of the users by capturing facial features. Enormous efforts have been made to develop reliable automated Facial Expression Recognition (FER) systems for use in machines and 
devices that are aware of the impact. These programs can more easily grasp the individual feelings and communicate with the consumer. Current technologies, however, are yet to achieve the maximum emotional and social capacities needed to create a rich and stable Human Machine Interaction (HMI). It is primarily due to the reality that HMI devices need to communicate with humans in an unregulated atmosphere (aka wild setting) where scene illumination, camera orientation, picture size, landscape, user-head posture, gender and ethnicity can differ considerably.

Furthermore, there are inadequate variations and annotated samples in the data that drive the creation of affective computing systems and particularly FER systems that can be used in developing these systems. Studies in late psychology showed that people basically convey their feelings externally. Study of facial expression is an essential part of genuinely rich Man-Machine Frameworks on communication (MMI), as it uses nonverbal signs all over to measure the user's enthusiasm. This work emphasises on creation of Neural Networks to detect user emotions and User engagement by utilising most commonly used datasets. In the recent literature and the work carried by various authors for user engagement detection, Convolutional Neural Networks (CNN) and Residual Networks (ResNet) are considerably used to improve the outcomes in emotional detections. Residual Networks utilizes skip networks that can connect between layers to enhance the learning pattern of the network. This rest of the paper is organised as follows, Section 2 describes the Related Work and Section 3 explains the Datasets. Section 4 explains the Residual Network. The details of experimentation are provided in section 5. The detailed Results are in Section 6. Finally, Section 7 concludes the paper.

\section{Related works}

In literature, there are several models to measure emotional behaviours: 1) definite models that select the emotion or affect from a list of categories of affectivity, including six specific emotions identified by Ekman etc. 2) Dimensional model where meaning, such as a valence and arousal, is selected over a sustained emotional scale 3) Facial Action coding systems, in the case of Action Units (AUs), all potential facial behaviours are identified. 4) Tagged Emotions, these are emotions that are grouped together based on Eckman's categorical model to create emotional tags as combinational outcomes [1]. The authors have grouped the emotions into four primary categories of learning environments: 1) boredom, 2) engaged, 3) frustrated and 4) confused Few authors have used combinational methods explicitly by combining two emotions that became unable to interpret mixed feelings into a limited collection of words sufficiently [2]. On the other hand, the dimensional model of affect can discern between slightly different displays of affect and represent minor changes in the intensity of each emotion on a continuous scale, such as valence and anticipation. Valence reflects how positive or negative an event is and excitement reflects whether an event is exciting, restless or calm.

In the continuous domain, dimensional perception of affect encompasses the strength and specific types of emotion. However, comparatively fewer studies have been performed to establish automatic algorithms for calculating affect using a cumulative dimensional model (e.g. valence and arousal). One of the key reasons for this is that building a massive database to cover the entire continuous space of valence and anticipation is costly and there are very few annotated face databases in the continuous domain. Facial Expression Recognition (FER) for different domains use supervised/semi supervised learning methods for automated affective computing. They require labelled dataset for training and testing, these datasets are generally created by subjects based on posed actions and also expressions extracted from videos enacted by various actors.

Recent studies of the education sectors have initiated to impart knowledge through online portals. These methods have become challenging in analysing the engagement levels of the students while teaching and learning is conducted through online portals [2]. Facial expression and affect datasets in the wild have been receiving a lot of attention recently. These datasets are either collected from movies or the world wide web and well labelled [35], and varied dimensions [6]. However, they cover just one model of affect, have a small range of subjects, or include little instances of certain emotions like disgust and sadness. A broad archive, with such a substantial quantity of object variations in wild condition covering numerous affect models is therefore, a requirement. Though there are several models for affect computing for emotional recognition in videos or single images, object localization and continuous emotional analysis has always been a challenging task due to face detection, posture recognition, segmentation, human pose, object association and for affective state classification using facial expressions in a cluttered environment. For the better growth of Massive Open Online Courses (MOOCs), there is a need to design smart interfaces that can simulate the interactions 
between the instructor and pupil. The principal disadvantage of existing e-learning systems is, that they cannot have direct input in real time Students (or instructors) during the delivery of the content, compared with traditional instruction in the classroom., MOOCs have a 91-93\% dropout rate [7]. Understanding user engagement at different junctures of the e-learning experience can help design intuitive interfaces that support students' better absorption of knowledge and personalize learning. The user 's understanding of affective state is an important computer vision sub-area, centred for a long time on datasets of the seven basic terms: neutral, happiness, sadness, anger, disgust, surprise and contempt [8]. In recent years, the data collection has been extended to cover successful states in terms of dimension representations [9-11], but the vast subtleties in affective states allow datasets for particular goals to be established. This strategy, which is backed by recent developments, including, tends to promote measurable outcomes. It has been found that in elearning and classroom settings students often prefer to communicate only a few effective conditions.

These included 7 fundamental emotions and a few emotions that are concentrated on learning. Distinct works focus on hand gesture [12], facial recognition, affective states, however there are a very few works on elaborating the available dataset on assessing various cognitive levels of understanding students emotional state for engagement and distraction. There are distinctive doubts on the curation and usage of facial data in facial recognition [13]. Few researchers have captured emotions under controlled environments while the subjects watch videos of different emotions [14-18]. With such methods being able to collect a vast number of frames, the variety of such repositories is restricted due to the number of participants, head orientation, and environmental exposures [21, 22].

Some of the works carried out on ISED datasets have predominantly used $\mathrm{CNN}$ as the crux, while some modifications on the network is incorporated to enhance the accuracy of the algorithm [25-26]. Feature extraction methods involving Local prominent directional patterns and local directional structural pattern have been used. However, these methods lack in efficient classification accuracy when compared to the CNN's [25-27]. Many authors use modified $\mathrm{CNN}$ in order to achieve greater results by adding multiple deep layers that enhance the performance of the system [29-31]. CNN's are prone to issues of vanishing gradients that leads to accuracy loss by curating the training into an expanding memory requirement. In all the major works carried out mentioned in this section, there is a need to improve the scope of detection rate and the precision of detection percentage for individual emotions. The need for a system that can reduce the error rates while training is important as the connections established in each layer kneads to weight updating and approximation to improve detection in the data.

This paper aims in analysing the Residual network's performance with respect to parameterized study that can establish the significance of classification model on three established datasets. Also, this work would compare the results with the existing models in terms of accuracy.

\section{Datasets}

Data for any work is pivotal and all the experiments are based on the data. There are critical datasets that emulate emotions and help in creating models for detecting emotions for various applications. Classification problems to detect emotions have recently been a field of study in various prominent datasets that contribute to the understanding of emotions. Though there are several datasets that help in analysing the face for emotions, there are few datasets for Indian Origin faces for a classroom environment. The learning environments not only are limited to the basic emotions also can be extended to various classes of classifications that can influence the accurate measurement of engagement in a class. This work focusses on elaborating the emotions by using the available dataset for Indian origin by combining basic classes, Engagement recognition and Learning centered emotions. The following datasets are used for this study are DAISEE, iSAFE and ISED databases. Table 1 lists the details of the available datasets for affectnet.

\section{The residual network}

In this work the priority is to elaborate the feature extraction process by creating a space where a particular emotion exposed by a human is discrete. This is achieved by extending the emotions into 10 classes and establishing a model that can eliminate the bias of learning and detection. network that was used for emotional analysis. This network is grouped as convolutional layers on the left of the figure and the Skip network on the right. The middle layers represent the connection between the residual connections and the convolution layers. SIU1CONV1 represents one single convolutional unit and S1U1BN1 represents the Batch normalization layer. Each of these layers are grouped into groups, each consisting of two convolutional and batch normalization layers. 
Table 1. Dataset details for facial emotional analysis

\begin{tabular}{|c|c|c|c|c|c|}
\hline $\begin{array}{l}\text { Sl } \\
\text { no }\end{array}$ & Name of the author & $\begin{array}{l}\text { Name of the } \\
\text { dataset }\end{array}$ & Database details & Affective states & $\begin{array}{l}\text { Emotions } \\
\text { enlisted }\end{array}$ \\
\hline 1 & Setty et al. [11] & IMFBD dataset & $\begin{array}{l}100 \text { movie } \\
\text { videos }\end{array}$ & Posed & $\begin{array}{l}\text { Fundamental } \\
\text { emotions }\end{array}$ \\
\hline 2 & Dhall et al. [14] & AFEW database & 957 videos & Temporal data & $\begin{array}{l}\text { Fundamental } \\
\text { emotions }\end{array}$ \\
\hline 3 & Happy et al. [15] & ISED database & $\begin{array}{l}428 \text { video data } \\
\text { from } 50 \\
\text { participants }\end{array}$ & $\begin{array}{l}\text { Collected from the } \\
\text { wild }\end{array}$ & $\begin{array}{l}\text { Fundamental } \\
\text { emotions }\end{array}$ \\
\hline 4 & Sapinski et al. [16] & $\begin{array}{l}\text { Multimodal } \\
\text { database }\end{array}$ & $\begin{array}{l}560 \text { images with } \\
16 \text { subjects }\end{array}$ & Learning centered & $\begin{array}{l}\text { Learning based } \\
\text { emotions }\end{array}$ \\
\hline 5 & Bian et al. [17] & $\begin{array}{l}\text { Spontaneous } \\
\text { expression database }\end{array}$ & $\begin{array}{l}30184 \text { images } \\
\text { from } 82 \text { students }\end{array}$ & Online learning & $\begin{array}{l}\text { Learning based } \\
\text { emotions }\end{array}$ \\
\hline 6 & Daisee et al [18] & DAISEE database & $\begin{array}{l}9068 \text { videos } \\
\text { from } 112 \text { users }\end{array}$ & $\begin{array}{l}\text { Engagement } \\
\text { recognition }\end{array}$ & $\begin{array}{l}\text { Learning based } \\
\text { emotions }\end{array}$ \\
\hline 7 & Lyons et al. [19] & JAFFE & $\begin{array}{l}7 \text { different } \\
\text { expressions } \\
\text { consisting of } 213 \\
\text { images }\end{array}$ & Acted expressions & $\begin{array}{l}\text { Fundamental } \\
\text { emotions }\end{array}$ \\
\hline 8 & Goodfellow I J et al. [20] & FER-2013 & 35685 images & $\begin{array}{l}\text { Collected from the } \\
\text { wild }\end{array}$ & $\begin{array}{l}\text { Fundamental } \\
\text { emotions }\end{array}$ \\
\hline 9 & Shivendra Singh et al. [21] & iSAFE & $\begin{array}{l}395 \text { videos from } \\
44 \text { volunteers }\end{array}$ & Acted expressions & $\begin{array}{l}\text { Fundamental } \\
\text { emotions }\end{array}$ \\
\hline 10 & Kaur et al. [22] & $\begin{array}{l}\text { Student } \\
\text { Engagement } \\
\text { Database } \\
\end{array}$ & $\begin{array}{l}78 \text { volunteers } \\
\text { with } 5 \text { mins } \\
\text { video }\end{array}$ & $\begin{array}{l}\text { Head pose and eye } \\
\text { Gaze }\end{array}$ & $\begin{array}{l}\text { Behavioural } \\
\text { Cues }\end{array}$ \\
\hline
\end{tabular}

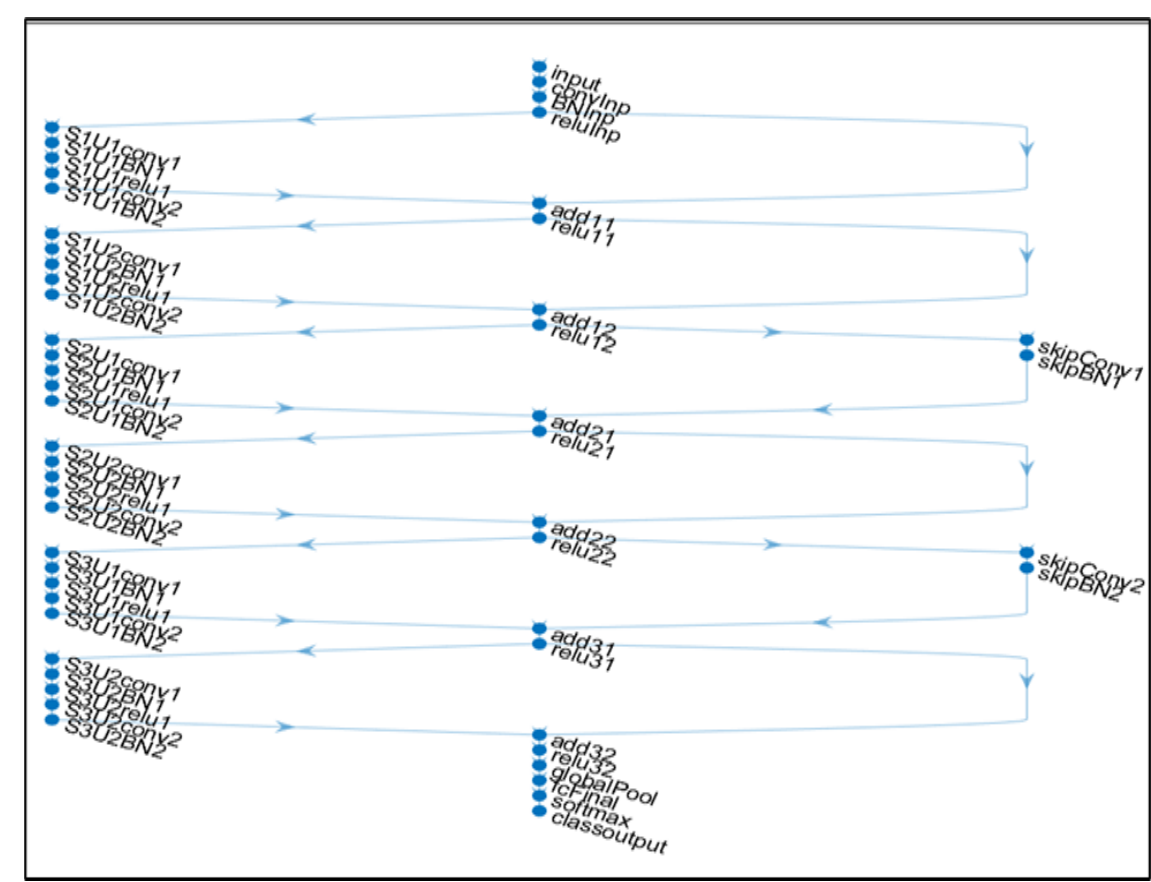

Figure. 1 The residual network

The residual connections are routed after the second batch into the third batch and similarly the second connection arises from the fourth batch into the fifth batch of layers. The ReLu layers are the interconnection layers providing the activation from the previous group of layers into the next group of layers. Additionally, the input layer has a convolution layer that channels the input images into the network. The final layers are equipped with pooling layer, SoftMax layer and the classification layer, the Pooling pools all the weights from the various distribution provided by various layers to help in dimensionality reduction and the SoftMax layer 


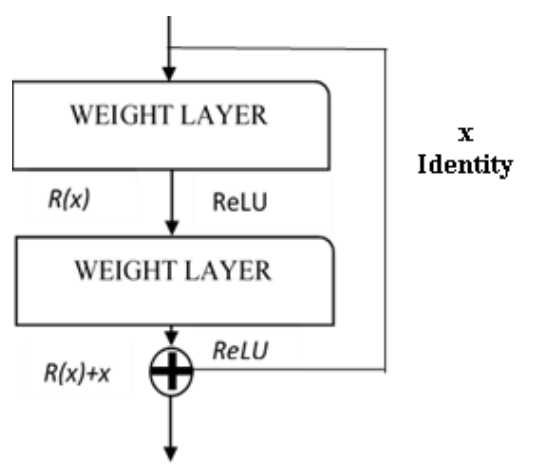

Figure. 2 A residual function

converts the weights into a normalized probability distribution. Based on the distribution the classification layer provides the class in which the image is classified.

Measuring learners' engagement by increasing scalability and appearance accessibility. The facial appearance training and extraction of the features are essential to enhance the understanding of the learner's engagement.

This work uses a light weight ResNet for feature extraction from the faces. Feature extraction and distinction are important tasks for analysing the emotions from the face. This work is divided into three phases i) Data Preparations ii) Emotion Understanding through residual Network iii) Validation of Results. The fundamental understanding of emotions lies in the orientation change in the face muscle. The facial muscles controlled by the facial nerve controls the emotions of an individual. The human brain transmits the data through the interconnections among the neurons that control the muscle movement to express. emotions.

CNN's generally perform well for shallow networks, as the network grows deeper depth wise vanishing gradient problem is quite obvious and also optimizing the data and parameters in the network are quite a tedious task CNN's, over the decade with deep layers have contributed to prominent results in the image processing arena. However, the issues like excessive training time, gradient vanishing due to deep networks and enormous parameters obtained while training are some persistent issues. Due to these reasons an upgraded model to especially keep the vanishing gradients under check is required and hence the Residual network was introduced. By connecting previous layers trained weights into the next layers using shortcut connections have achieved greater impact in improving the accuracy and reducing time taken for training. In this work a lightweight network using ResNets is used. Fig. 1 explains the model that is used to learn meaningful interpretations from images. Every residual block has a $3 \times 3$ convolution layer followed by a batch normalisation layer followed by a ReLU activation feature. This is further accompanied by a $3 \times 3$ convolution layer and a batch normalisation layer. The skip link skips both of these layers and attaches immediately before the ReLU activation feature. These residual blocks are replicated in order to form a residual network. Deep networks are prone to degradation problems due to the Vanishing gradients and fitting issues that leads to worse training errors. Deep networks need not be "harder" to match intuitively If there are such layers $\mathrm{N}$ with maximum data precision, then the identity-mapping layers $M(x)$, following $\mathrm{N}$ can only be learned by mapping the layer to be learned; the network would have efficient $N$ layer performance.

However, it is not simple to drive weights in such a way that they exactly yield identity mapping. The idea of residual learning leads to a residual function $R(x)-x$. This is interpreted as a stack of layers that computes the mapping as $y=R(x)+x$ as shown in Fig.2 To learn $\mathrm{y}=\mathrm{R}(\mathrm{x})$, the learning can happen directly through $\mathrm{F}(\mathrm{x})$ where,

$$
F(x)=R(x)-x
$$

therefore, our underlying mapping is $y=M(x)$, such that the network learns the added weights from the previous layer, where $y$ is represented as $F(x)+x$,

$$
y=R(x)-x+x
$$

Identity mapping is made convenient due to the introduction of allocating all the weights to 0 , so that $R(x)=0$ and $F(x)=-x$ such that $y=x$ is trained. ResNet is defined by its building block and it is denoted by,

$$
y=\left(x_{i}, W_{i}\right)+x
$$

where, F can be multiple layers. A shortcut operation and element-wise addition are done by the '+' operation as seen in Fig. 2. New parameters are not added by the shortcut link in the network and hence training the network in this way does not increase training time due to the number of parameters that must be trained. But if dimensionalities are distinct, this is not feasible.

To handle dimensionality approximation, a projection matrix $W_{s}$ that is associated with $x$ on the same space $F(x)$. Hence, Eq. (3) can be modified as,

$$
y=F\left(x,\left\{W_{i}\right\}\right)+W_{s} x
$$

Degradation of gradients can be controlled by adding the identity mapping coefficients and $W s$ is used for 
matching the dimensions of the previous layer with the next layer. Multiple convolutional layers are represented with the function $F(x,\{W i\})$. The element-wise addition is performed on two feature maps, channel by channel. Table. 2 illustrates the details of various parameter setting used in the network. Eq. (5), explains the CNN process, in which: $n$ is the size of the image, $p$ is the padding size, $f$ is the size of the filter, $n c$ is the number of channels, $n f$ is the number of filters and $s$ is the stride. convolutions are carried out on each image based on $n, n c$ and $f$, preserving the relation between pixels and creating a matrix of feature maps. $s$ is used to shift the filter over the image pixels $p$.

$$
[n, n, n c] .[f, f, n c]=\left[\left[\frac{n+2 p-f}{s}+1\right]\left[\frac{n+2 p-f}{s}+1\right], n f\right]
$$

After two layers of convolution Eq. (4) is introduced as a shortcut layer to carry information from the previous layers into the next layer. The residual connections help in associating the prediction values that were estimated by the previous layers as an input into the next layer. The residual function computes and matches the actual value with the predicted value. If the value of $x$ is equal to the actual value then the residual function is zero resulting in a higher derivative. along with the residual connections batch normalization is also carried out in the block to normalize the values to a threshold where the derivatives are not too small to be removed due to least significance. All the layers, parameters mentioned are the outcome of OHP. This tuning helps in assembling the required number of layers to extract meaningful interpretations of mid-level and highlevel features after each iteration to create a pool of weighted probabilities. These probabilities are used to classify the images during validation and testing.

\section{Experimentation}

The Residual Network was used on the following datasets, ISED, iSAFE and Daisee Datasets. The network is trained with images of the faces of Indian origin. Since these images are created by the authors for an E-learning environment the same trained network was used to create an observation on the Online classes that are being conducted during these pandemic years. All the experiments were carried out using Intel Xeon E3 based workstations, NVIDIA Gforce GTX graphics card on a 32 GB RAM and Matlab2019b was used as the platform to train and validate the network. Images from the testing data is drawn at random and fed into the network, these
Table 1. Parameter setting detail

\begin{tabular}{|l|l|}
\hline Parameter's Name & Value \\
\hline Networks Layers & 50 \\
\hline InitialLearnRate(Lr) & $1.00 \mathrm{E}-04$ \\
\hline Regularization Function & $1_{2}$ Regularization \\
\hline GradientThreshold & '12norm' \\
\hline MaxEpochs & 40 \\
\hline MiniBatchSize $\left(B_{s}\right)$ & 32 \\
\hline Verbose Frequency & 50 \\
\hline Validation Frequency $\left(\mathrm{V}_{\mathrm{f}}\right)$ & 500 \\
\hline Shuffle & 'every-epoch' \\
\hline Padding Direction & 'right' $(1,1)$ \\
\hline Filter Size & $(3 \times 3)$ \\
\hline Stride & $(1,1)$ \\
\hline Number of Filters $($ layer $)$ & $8,16,32,64$ \\
\hline Optimiser & SGDM \\
\hline Learning Rate Scheduler & Piecewise \\
\hline Image size & $192 \times 192 \times 3$ \\
\hline Learning Droprate & 60 iterations \\
\hline
\end{tabular}

images are tested for True Positive, false positive, True negative or false. The images are resized and fed into the Residual network with the sizes of $128 \mathrm{x}$ $128 \times 3$ dimensions.

Images from each dataset for training are 508 images from ISED and iSAFE datasets that trains data for 7 classes. Similarly, 5295 images are used for training data from Daisee dataset. The network was created from the scratch and the network was used to improve the efficiency of emotional understanding. However, every time training is carried out on the datasets, the learning rate of the network is set to $1.00 \mathrm{E}-04$ this helps the network to learn features from the inception. The network uses a piecewise learning rate scheduler, this enhances the learning rate by decreasing the learning rate often and optimizing the network for a higher degree of weight vector distribution. The data is shuffled after each epoch and the mini batch size was fixed at 32. Fig. 2 elicits the details of the network where two skip networks are introduced. The plain network had 40 layers, while the introduction of the residual layers has increased the number of layers to 50 .

The number of layers is still lesser than the prominent networks like ResNet 32, ResNet50 and Resnet 101. The pooling layers help in reducing the dimensionality of the features extracted, while the $\mathrm{ReLu}$ layers were used in the network as activation functions. The layers were not chosen at random, however the layers were precisely placed after several iterations and changes to the entire network based on the performances based on OHP. Deep Networks with higher number of layers and residual networks and hence achieving the desired results for the data took a long time to reach an optimum 


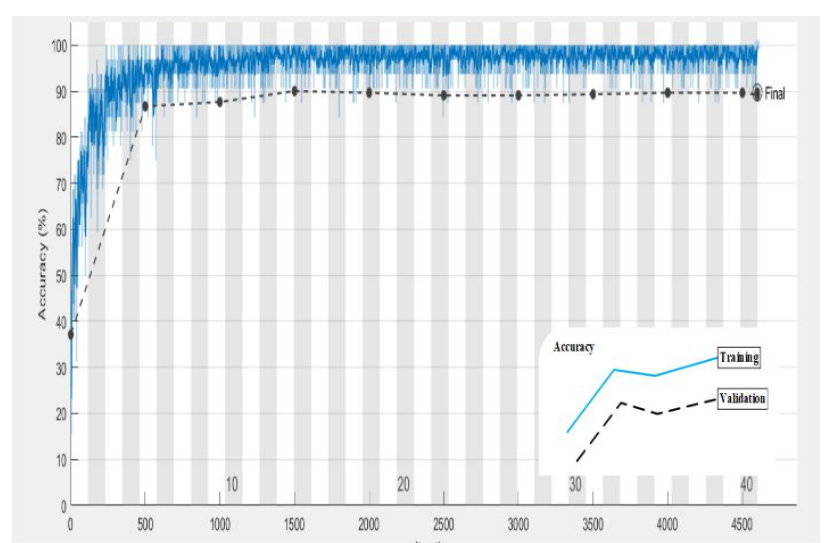

Figure. 3 Training \& validation accuracy for daisee dataset

solution and also the training accuracy was lesser than $70 \%$. This was due to the increase in feature pooling requirement and gradient descent issue was a common problem.

The intent is to create this optimized network for emotional understanding through multiple classes. The data available is imbalanced and hence F1 score is used to determine the efficiency of the algorithm. The training data validation was conducted after 500 iterations, that provide the information about the training progress, parallelly once the loss function was also measured. The details of the training are provided in Fig. 3. The data available is imbalanced hence F1 score is used to determine the efficiency of the algorithm. The training data validation was conducted after 500 iterations, which provides the information about the training progress, parallelly the loss function was also measured. The details of the training are provided in Fig. 3. The Stochastic Gradient Descent for the Function at $x$ is,

$$
\nabla f(x)=\frac{1}{n} \sum_{i=1}^{n} \nabla f_{i}(x)
$$

Stochastic gradient descent (SGD) reduces computational cost at each iteration for an unbiased estimate of the gradient $\nabla f(x)$. At each iteration the SGD is uniformly sampled to an index of $i \in\{1, \ldots, n\}$ at random, and gradient $\nabla f_{i}(x)$ is computed to update $\mathrm{x}$ :

$$
x \leftarrow\left\lfloor x-\eta \nabla f_{i}(x)\right]
$$

Replacing $\eta$ with a learning rate $\eta(t)$ that is time dependent improves in controlling the convergence rate of an algorithm that should produce optimized outcomes. The piecewise constant for a time-based SGD is defined as in Eq. (8). $\eta(t)$ reduces the learning rate whenever the progress towards optimization is not improving.

$$
\eta(t)=\eta_{i} \text { if } t_{i} \leq t \leq t_{i+1}
$$

Eq. (9) is used to calculate the efficiency of the classifier based on the confusion matrix generated based on the classifier on the test data. Kappa Statistics is a measure that calculates how closely the instances are classified by the algorithm that matched the data labelled as ground truth also controlling the accuracy of a random classifier as measured by the expected accuracy.

$$
\operatorname{Kappa}(\kappa)=\frac{p_{o-} p_{e}}{1-p_{e}}
$$

Where, $p_{o}$ is the observed agreement, and $p_{e}$ is the expected agreement. It recommends the performances of the classifier over a classification that is generated merely based on a guess at random according to the frequency of each class. Accuracy, Precision, Recall, Sensitivity, Specificity and F1 score are used as metrics gauge the performance of the classifier for each class on the three datasets. The iSAFE and ISED datasets are combined together for 7 classes as a single database and the Daisee Dataset uses 4 classes. Cohens-Kappa is also used to measure the efficiency of the classifier as it is a multiclass and imbalanced data.

\section{Results and discussions}

The training samples are shuffled and taken at random after every epoch to reduce over-fitting or under-fitting issues. The Training Accuracy(T) and Validation Accuracy $(\mathrm{V})$ is plotted and visualized for the efficiency index of the model after every iteration, Fig. 3, illustrates the training and validation results acquired using the Residual Network model. The Validation frequency $(V f)$ is an important parameter as it tests the model's training efficiency in regular intervals. The value for $V_{f}$, is set for every 500 iterations for this model. A weighted average of the scores independently derived from individual layers using the posterior class probabilities is cumulated to improve Learning rate and reduce Validation Loss.

These weights are then trained for face image on a cross dataset, which helps in reducing the blindness of the model to newer data. However, while comparing with the other methods as mentioned in Table 3, Emonet performs with an accuracy higher than the majority of the work carried out earlier. In many of the dataset's individual classifications, there have been instances where emotions have been wrongly classified, however individualistic and 


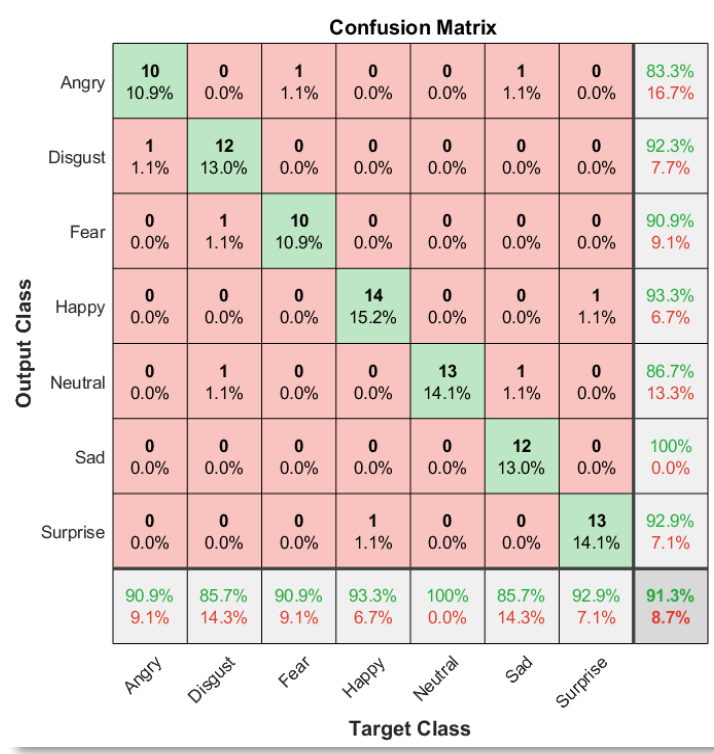

Figure. 4 Confusion matrix for ISED \& iSAFE dataset

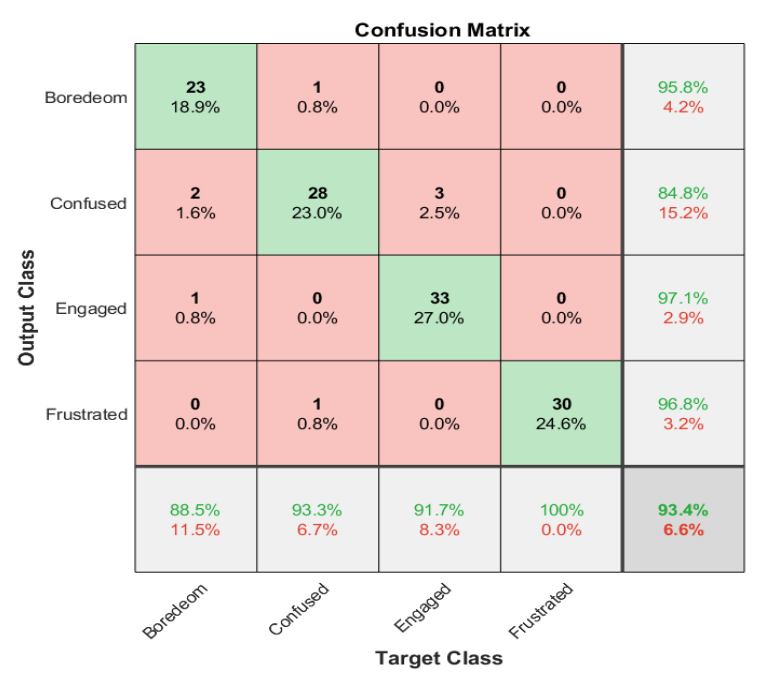

Figure. 5 Confusion matrix for daisee dataset

holistic approach has enhanced the model's performance. To shed light on this classification model the following metrics Accuracy, Precision, Recall, Specificity and F1-score is calculated for EmoNet on the dataset and the results are furnished in Table 3. The number of epochs used to train the network was set to 40. The network has undergone numerous trial and error methods to fix on the hyperparameters. Moreover, decisive conclusions in the number of iterations were based on the Loss function. Cross validation was performed on the dataset, where the data was split into $70 \%$ training and $30 \%$ validation data. The progress of the network to evaluate overfitting. At each $V_{f}$ the network validates the data for validation. The network is
Table 2. Metric table

\begin{tabular}{|c|c|c|c|c|c|c|}
\hline Data & Class & Acc & Pre & Sen & Spe & F1 \\
\hline \multirow{7}{*}{ ISED } & Anger & 95.55 & 0.91 & 0.83 & 0.99 & 0.87 \\
\hline & Disgust & 96.53 & 0.86 & 0.92 & 0.97 & 0.89 \\
\hline & Fear & 95.63 & 0.91 & 0.91 & 0.99 & 0.91 \\
\hline & Happy & 97.67 & 0.93 & 0.93 & 0.99 & 0.93 \\
\hline & Neutral & 98.03 & 1.00 & 0.87 & 1.00 & 0.93 \\
\hline & Sad & 94.34 & 0.86 & 1.00 & 0.97 & 0.92 \\
\hline & Surprise & 96.67 & 0.93 & 0.93 & 0.99 & 0.93 \\
\hline \multirow{4}{*}{ Daisee } & Boredom & 96.61 & 0.88 & 0.96 & 0.97 & 0.92 \\
\hline & Confusion & 94.21 & 0.93 & 0.85 & 0.98 & 0.89 \\
\hline & Engaged & 96.61 & 0.92 & 0.97 & 0.96 & 0.94 \\
\hline & Frustrated & 99.13 & 1.00 & 0.97 & 1.00 & 0.98 \\
\hline
\end{tabular}

consistent and there is a close correlation between the validation accuracy and training accuracy. Based on this training the model achieved $84.97 \%$ validation accuracy on the Daisee dataset and $88.76 \%$ on the ISED \& iSafe dataset combined. Residual connections establish a prominent learning framework for the emotions in these datasets.

Fig. 4 and Fig. 5 provides the details of the confusion matrix $\left(\mathrm{C}_{\mathrm{M}}\right)$ acquired over the two datasets and are derived as an outcome of the classifier from the images that were not used while training the network. In addition to the $\mathrm{C}_{\mathrm{M}}$, of each classes the accuracy, precision, recall, sensitivity and F1 score are calculated. The results prove that the residual connections introduced to the CNN's have created a vital impact in improving the outcomes for detecting and recognizing emotions. The matrix also displays the percentage of False Positives and False Negatives for each class that provides a deep understanding of the performance for each class in the data.

Table. 3 displays the findings obtained using the ISED, iSafe and Daisee database from the different models used for emotional identification. Compared to recent works using $\mathrm{CNN}$ and conventional methods of definition, the new model has been effective. Though the metric F1- measure is used to measure the performance of the classifier, it is the harmonic mean of precision and recall. These metrics apart from using the conventional methods of analysing accuracy from the classification data, it also elicits the performance of the classifiers with different data. This model is also compared with the traditional CNN model with 40 layers and the accuracy on ISED data was recorded as $90.53 \%$ and with the iSAFE database at $91.78 \%$. Improvement in the results were found while using residual functions to the existing EMONET model.

The proposed model has a good ranking score based on Table. 3. While all of the other models use 
Table 4. Performance comparison of various available models

\begin{tabular}{|c|c|c|c|}
\hline $\begin{array}{l}\text { Sl } \\
\text { no }\end{array}$ & Name of the Method & Dataset & $\begin{array}{l}\text { Acc } \\
(\%)\end{array}$ \\
\hline 1 & CNN [23] & ISED & 51.6 \\
\hline 2 & CNN [23] & ISED & 59.3 \\
\hline 3 & Inception V3 [23] & ISED & 47.9 \\
\hline 4 & EmotionNet 2 [23] & ISED & 21.0 \\
\hline 5 & EmotionalDAN [23] & ISED & 62.0 \\
\hline 6 & CNN [24] & ISED & 82.9 \\
\hline 7 & $\begin{array}{cl}\text { Local prominent } \\
\text { directional patterns [25] } \\
\text { i. } & \text { LBP (Local } \\
\text { ii. } & \text { Binary Pattern) } \\
& \text { LDP (Local } \\
& \text { Directional } \\
\text { iii. } & \text { Pattern) } \\
& \text { LDN (Local } \\
& \text { Directional } \\
& \text { Number) } \\
\text { iv. } & \text { LPTP (Local } \\
& \text { Directional } \\
& \text { Ternary Pattern) } \\
\text { v. } & \text { PTP (Positional } \\
& \text { Ternary Pattern) } \\
\text { vi. } & \text { HOG (Histogram } \\
& \text { Of Gradients) } \\
\text { vii. } & \text { LPDP (Local } \\
& \text { Prominent } \\
& \text { Directional } \\
& \text { Pattern) } \\
\text { viii. } & \text { LPDP } f(\text { Local } \\
& \text { Prominent } \\
& \text { Directional } \\
& \text { Pattern } f \text { ) } \\
\end{array}$ & ISED & $\begin{array}{l}76.75 \\
77.80 \\
78.32\end{array}$ \\
\hline 8 & Landmark Detection [26] & ISED & 34 \\
\hline 9 & $\begin{array}{l}\text { Local directional-structural } \\
\text { pattern [27] }\end{array}$ & ISED & 77.78 \\
\hline 10 & LDP+KPCA [28] & Daisee & 90.89 \\
\hline 11 & Hybrid CNN [29] & Daisee & 86 \\
\hline 12 & $\begin{array}{l}\text { Deep Engagement } \\
\text { Recognition Network [30] }\end{array}$ & Daisee & 57.9 \\
\hline 13 & $\begin{array}{l}\text { Very Deep Convolutional } \\
\text { Network [31] }\end{array}$ & Daisee & 92.33 \\
\hline 14 & Proposed Model & $\begin{array}{l}\text { ISED \& } \\
\text { iSAFE }\end{array}$ & 91.3 \\
\hline 15 & Proposed Model & Daisee & 93.44 \\
\hline
\end{tabular}

state-of-the-art deep learning networks and traditional methods such as Inception V3, CNN, and the well-known local directional patterns. The model proposed exceeds the precision of all ISED algorithm models that have used the database. Conventional methods, CNN, Hybrid CNN's and many more recent works that have been carried out in the recent years are prominent in enhancing the efficiency of emotional analysis especially in the field of student engagement and attention estimation. Based on Eq. (9) the classifiers accuracy on both the dataset is calculated based on the confusion matrix. From Table. 3 , various parametric results show that the network was able to achieve significant outcome using the residual network. The average results for the dataset ISED and iSafe dataset are, Accuracy 91.3\%, Error at $8.7 \%$, Sensitivity $91.34 \%$, Specificity 98.56, precision $91.35 \%$, false Positive rate is recorded at $1.44 \%$ and the Kappa's Coefficient 0.65 which is recorded to be a substantial classifier for the database. Similarly, in the Daisee dataset, overall results for Accuracy: $93.44 \%$, Error rate: $6.56 \%$, Sensitivity: 93.63\%, Specificity: $97.82 \%$, Precision $93.37 \%$, false positive rate is recorded at $2.18 \%$ and the Kappa's Coefficient 0.825 which is recorded to be a perfect classifier for the database. The proposed method has attained promising results due to the enormous images used for training, optimised layers to enrich the shallow network to learn, understand and capture features. The Features learned using the Network makes it sophisticated for classification with higher accuracy. The Feature maps created after each layer that uses the activation functions enhances the network by reducing the vanishing gradient and overfitting issues that are prominent in the traditional $\mathrm{CNN}$. The network is compared with the results that are deeper and most commonly used trained networks. Table. 4, enlists the methods used by various authors and the accuracy that it has achieved on the dataset, though there are different classes in each of the datasets our model has attained a signific-ant improvement in all parameters used to measure the tangibility in the introduction of residual networks on conventional CNN.

There are three observations about the network's performance, firstly degradation issues have rapidly decreased due to the lower training error rate which was observed to be at 0.265 . The reduced training error improves the efficiency of the learning due to the optimum depth of the network. Secondly, the identity connections as mentioned in Fig. 1, have helped in significantly decreased the time complexity for training and validation by $30 \%$. Thirdly, the network uses SGD solver and it is able to find good solutions. Though the network is shallow, gradient descent algorithm works on batches of smaller sizes this enables the network to train on smaller batches and create multiple layers of features. These features are the crux of the classification unit to create probabilities on the Weighted layers by accurately turning on the exact neurons to provide precise and accurate results. Two-Fold Cross validations provide visibility on the network's validation outcome during 
the training phase. This provides a closer detail of the performance of the network. Incorporating Optimized Hyper Parametric Settings that are discussed in Section. 4, has cushioned the network to be customized for better performance.

\section{Conclusions}

The proposed work intends to evaluate the performance of the inclusion of residual layers to an existing CNN model. CNN models are prone to vanishing gradients and loss in accuracy as the networks grow deep. The residual connections on the shallow network is designed from the scratch specifically for the purpose of detecting students' engagement on an E-Learning platform. Both behavioral classes like boredom, engaged, frustrated and confusion classes along with emotional classes are considered in this work. This work utilizes students' facial features to predict and classify images in the wild to calculate the accuracy of the proposed approach. In this model, the network uses residual networks to improve connections from previous layers into the next layers to improve the learning and classification response of the system. Two-fold cross validations are used to understand the capability of the model. This network is trained on three Indian Datasets indigenously, to be able to detect the emotional and behavioral intent of the students. The total layers used in the network is 50 . The shallow network helps in efficient learning model that is able to validate images at an average of $86.87 \%$ during training. The model is tested for detection efficiency with test data and compared with state-of-the-art models that are built with $\mathrm{CNN}$ as primary network. The usage of Residual Connections and Optimized Hyper Parametric Settings has considerably enhanced the performance in creating and using the network for a Indian face based emotional classification model.

The Furnished results in Table. 3 and Table 4 based on Fig. 4 and Fig. 5 show that the model outperforms the other state of the art techniques. Also, the classifiers performance is evaluated with the help of Kappa score and the network performs well and the classifier is diligently able to perform at $82.51 \%$. The proposed model is evaluated for performance measures using standard evaluation metrics that results in improvement close to $2 \%$ in various parameters. The proposed model will be tested for group engagement detections and evaluate the Valence and Arousal of the group using the model as a future enhancement.

\section{Conflicts of Interest}

The authors declare no conflict of interest.

\section{Author Contributions}

The contributions by the authors for this research article are as follows: "conceptualization, methodology, Formal analysis and writing — original draft preparation Michael Moses Thiruthuvanathan; Result validation, resources, formal analysis writing-review and editing and supervision Balachandran Krishnan; data curation, Result Validation and ethical inference Madhavi Rangaswamy"

\section{Acknowledgments}

Authors wish to acknowledge the technical and infrastructure help rendered by the faculty members from the department of Computer Science and Engineering, CHRIST (Deemed to be University), Bangalore, India.

\section{References}

[1] A. Mollahosseini, B. Hasani, and M. H. Mahoor, "AffectNet: A Database for Facial Expression, Valence, and Arousal Computing in the Wild", IEEE Transactions on Affective Computing, Vol. 10, No. 1, pp. 18-31, 2019.

[2] T. Ashwin and R. Guddeti, "Affective Database for E-Learning and Classroom Environments using Indian Students' Faces, Hand Gestures and Body Postures", Future Generation Computer Systems, Vol. 108, No. 1, pp. 334-348, 2020.

[3] A. Dhall, R. Goecke, J. Joshi, M. Wagner, and T. Gedeon, "Emotion Recognition in the Wild Challenge 2013", In: Proc. of $15^{\text {th }}$ International Conf. on Multimodal Interaction, ACM, pp. 509-516, 2013.

[4] I. Goodfellow, D. Erhan, P. L. Carrier, A. Courville, M. Mirza, B. Hamner, W. Cukierski, Y. Tang, D. Thaler, and H. Lee, "Challenges in Representation Learning: A Report on Three Machine Learning Contests", In: Proc. of International Conf. on Neural Information Processing, Vol. 64, No. 1, pp. 59-63, 2015.

[5] A. Mollahosseini, B. Hasani, M. J. Salvador, H. Abdollahi, D. Chan, and M. H. Mahoor, "Facial Expression Recognition from World Wild Web", In: Proc. of IEEE Conf. on Computer Vision and Pattern Recognition (CVPR) Workshops, Vol. 1, pp. 168-195, 2016.

[6] S. Zafeiriou, A. Papaioannou, I. Kotsia, M. Nicolaou and G. Zhao, "Facial Affect "In-the- 
Wild": A Survey and a New Database", In: Proc. of IEEE Conf. on Computer Vision and Pattern Recognition Workshops (CVPRW), pp. 14871498, 2016.

[7] L. Rothkrantz, "Dropout Rates of Regular Courses and MOOCs", In: Proc. of International Conf. on Computer Supported Education, Rome, pp. 25-46, 2016.

[8] M. Li, H. Xu, X. Huang, Z. Song, X. Liu and X. $\mathrm{Li}$, "Facial Expression Recognition with Identity and Emotion Joint Learning", IEEE Transactions on Affective Computing, Vol. 4, No. 8, pp. 411-416, 2018.

[9] S. M. Mavadati, M. H. Mahoor, K. Bartlett, P. Trinh, and J. F. Cohn, "Disfa: A Spontaneous Facial Action Intensity Database", IEEE Transactions on Affective Computing, Vol. 4, No. 2, pp. 151-160, 2013.

[10] T. Ashwin and R. Guddeti, "Unobtrusive Students' Engagement Analysis in Computer Science Laboratory Using Deep Learning Techniques", In: Proc. of IEEE 18th International Conf. on Advanced Learning Technologies (ICALT), pp. 436-440, 2018.

[11] S. Setty, M. Husain, P. Beham, J. Gudavalli, M. Kandasamy, R. Vaddi, V.Hemadri, J. Karure, R. Raju, and B. Rajan, "Indian Movie Face Database: A Benchmark for Face Recognition under Wide Variations", In: Proc. of Fourth National Conf. on Computer Vision, Pattern Recognition, Image Processing and Graphics (NCVPRIPG), IEEE, 2013, pp. 1-5, 2013.

[12] S. Patwardhan and G. M. Knapp, "Affect Intensity Estimation Using Multiple Modalities", In: Proc. of Florida Artificial Intelligence Research Society Conf., pp. 130-133, 2014.

[13] R. Noorden, "The ethical questions that haunt facial-recognition research", Nature. Vol. 587: pp. 354-358, 2020.

[14] A. Dhall, R. Goecke, S. Lucey, and T. Gedeon, "Collecting Large, Richly Annotated FacialExpression Databases from Movies", IEEE MultiMedia, Vol. 19, No. 3, pp. 34-41, 2012.

[15] S. Happy, P. Patnaik, A. Routray, and R. Guha, "The Indian Spontaneous Expression Database for Emotion Recognition", IEEE Transactions on Affective Computing, Vol. 8, No. 1, pp. 131142, 2017.

[16] T.Sapinski, D. Kaminska, A. Pelikant, C. Ozcinar, E.Avots, and G. Anbarjafari. "Multimodal Database of Emotional Speech, Video and Gestures", In: Proc. of International Conf. on Pattern Recognition Information Forensics, pp. 153-163, 2018.
[17] C. Bian, Y. Zhang, F. Yang, W. Bi, and W. Lu, "Spontaneous Facial Expression Database for Academic Emotion Inference in Online Learning", IET Computer Vision. Vol. 13, No. 3, pp. 329-337, 2018.

[18] A. Gupta, A. D'Cunha, K. Awasthi, and V. Balasubramanian, "Daisee: Towards User Engagement Recognition in the Wild", arXiv preprint arXiv: 1609.01885, 2016.

[19] M. J. Lyons, S. Akamatsu, M. Kamachi, J. Gyoba, J. Budynek, "The Japanese female facial expression (JAFFE) database", In: Proc. of Third International Conf. on Automatic Face and Gesture Recognition, pp. 14-16, 1998.

[20] I. Goodfellow, D. Erhan, P. L. Carrier, A. Courville, M. Mirza, B. Hamner, W. Cukierski, Y. Tang, D. Thaler, D.-H. Lee, Y. Zhou, C. Ramaiah, F. Feng, R. Li, X. Wang, D. Athanasakis, J. Shawe-Taylor, M. Milakov, J. Park, R. Ionescu, M. Popescu, C. Grozea, J. Bergstra, J. Xie, L. Romaszko, B. Xu, Z. Chuang, and $\mathrm{Y}$. Bengio. "Challenges in representation learning: A report on three machine learning contests", Neural Information Processing, Vol. 8228, pp. 117-124, 2013.

[21] S. Singh and S. Benedict, "Indian Semi-Acted Facial Expression (iSAFE) Dataset for Human Emotions Recognition", Advances in Signal Processing and Intelligent Recognition Systems. SIRS Communications in Computer and Information Science, Vol 1209, No. 1, pp. 150$162,2019$.

[22] A. Kaur, A. Mustafa, L. Mehta and A. Dhall, "Prediction and Localization of Student Engagement in the Wild", Digital Image Computing: Techniques and Applications (DICTA), Canberra, Australia, pp. 1-8, 2018.

[23] I. Tautkute, T. Trzcinski, and A. Bielski, "I Know How You Feel: Emotion Recognition with Facial Landmarks", In: Proc. of IEEE Conf. on Computer Vision and Pattern Recognition Workshops (CVPRW), pp. 1959-1974, 2018.

[24] S. Gonzalez-Lozoya, J. Calleja and L. Pellegrin, H.Escalante, Ma. Medina and A. Benitez-Ruiz, "Recognition of Facial Expressions based on CNN Features", Multimedia Tools \& Application, Vol. 79, pp. 13987-14007, 2020.

[25] F. Makhmudkhujaev, M. Abdullah-Al-Wadud, M. Iqbal, B. Ryu, and O. Chae, "Facial Expression Recognition with Local Prominent Directional Pattern", Signal Processing: Image Communication, Vol. 74, No. 1, pp, 1-12, 2019.

[26] S. Engoor, S. SendhilKumar, C. Hepsibah Sharon, and G. S. Mahalakshmi, "Occlusionaware Dynamic Human Emotion Recognition 
Using Landmark Detection", In: Proc. of 6th International Conf. on Advanced Computing and Communication Systems (ICACCS), pp. 795-799, 2020.

[27] A. Rivera, J. Rojas Castillo, and O. Oksam Chae, "Local Directional Number Pattern for Face Analysis: Face and Expression Recognition", IEEE Transactions on Image Processing, Vol. 22, No. 5, pp. 1740-1752, 2013.

[28] M. Dewan, F. Lin, D. Wen, M. Murshed and Z. Uddin, "A Deep Learning Approach to Detecting Engagement of Online Learners", In: Proc. of IEEE Smart World, Ubiquitous Intelligence \& Computing, Advanced \& Trusted Computing, Scalable Computing \& Communications, Cloud \& Big Data Computing, Internet of People and Smart City Innovation, pp. 1895-1902, 2018.

[29] T. Ashwin and R. Guddeti, "Automatic detection of students' affective states in classroom environment using hybrid convolutional neural networks", Education Information Technologies, Vol. 25, No. 1, pp. 1387-1415, 2020.

[30] O. Nezami, M. Dras, L. Hamey, D. Richards, S. Wan, and C. Paris, "Automatic Recognition of Student Engagement Using Deep Learning and Facial Expression", In: Proc. of Machine Learning and Knowledge Discovery in Databases, Lecture Notes in Artificial Intelligence, Springer, Part III, Vol. 11908, pp. 273-289, 2020.

[31] T. Huang, Y. Mei, H. Zhang, S. Liu and H. Yang, "Fine-grained Engagement Recognition in Online Learning Environment", In: Proc. of IEEE 9th International Conf. on Electronics Information and Emergency Communication (ICEIEC), Beijing, China, pp. 338-341, 2019. 